\title{
The cosmological background of vector modes
}

\author{
Teresa Hui-Ching $\mathrm{Lu}^{1}{ }^{*}$ Kishore Ananda ${ }^{1}$, Chris Clarkson ${ }^{1}$, and Roy Maartens ${ }^{2}$ \\ 1 Cosmology $\& 3$ Gravity Group, Department of Mathematics and Applied Mathematics, \\ University of Cape Town, Rondebosch 7701, Cape Town, South Africa \\ 2 Institute of Cosmology and Gravitation, University of Portsmouth, \\ Dennis Sciama Building, Burnaby Road, Portsmouth PO1 3FX, UK
}

(Dated: November 1, 2018)

\begin{abstract}
We investigate the spectrum of vector modes today which is generated at second order by density perturbations. The vector mode background that is generated by structure formation is small but in principle it contributes to the integrated Sachs-Wolfe effect, to redshift-space distortions and to weak lensing. We recover, clarify and extend previous results, and explain carefully why no vorticity is generated in the fluid at second order. The amplitude of the induced vector mode in the metric is around $1 \%$ that of the first-order scalars on small scales. We also calculate the power spectrum and the energy density of the vector part of the shear at second order.
\end{abstract}

\section{INTRODUCTION}

Cosmological vector perturbations at linear order satisfy an evolution equation and a momentum constraint equation which can be found by calculating the $i-j$ and $0-i$ parts of the Einstein field equations respectively. In the case of a perfect fluid (or scalar field) there is no source in the vector evolution equation and its solutions decay as $1 / a^{2}$. In the standard models of structure formation, inflation does not generate vector perturbations, and we can therefore ignore vector perturbations at first order. However, vector perturbations will be generated at nonlinear order by the growth of density perturbations. Here we revisit the analysis of this cosmological vector background (see Refs. [1, 2, 3, 4, 5, 6, 7, for previous work). As we show below, the amplitude of the induced vectors is surprisingly large on small scales, when compared to the amplitude of the first-order Newtonian potential. In order to investigate whether this is important dynamically we calculate the vector part of the shear and compare it to the usual first-order scalar shear.

We consider perturbations of a flat Robertson-Walker background up to second order: $g_{\mu \nu}=\bar{g}_{\mu \nu}+\delta^{(1)} g_{\mu \nu}+\delta^{(2)} g_{\mu \nu}$. At first order, $\delta^{(1)} g_{\mu \nu}$ only contains scalar perturbations: we neglect the tensor perturbations, and vector perturbations are not generated at first order in the standard model. For the second-order perturbations, $\delta^{(2)} g_{\mu \nu}$, we project out the vector modes. In the Poisson gauge [1]

$$
d s^{2}=-a^{2}\left[1+2 \Phi_{(1)}\right] d \eta^{2}-a^{2} S_{i}^{(2)} d x^{i} d \eta+a^{2}\left[1-2 \Phi_{(1)}\right] d \vec{x}^{2},
$$

where $\Phi_{(1)}$ is the first-order Newtonian potential (we assume zero scalar anisotropic stress at first order), and $S_{(2)}^{i}$ describes the gauge-invariant second-order vector modes, so that $\partial_{i} S_{(2)}^{i}=0$. We effectively ignore the second-order scalar mode $\Phi_{(2)}$ since we are interested only in the second-order vector modes: second-order modes can be consistently split into scalar and vector, but the equations for the second-order vector modes will contain source terms that are quadratic in the first-order scalar modes. In what follows we will drop the order indices when there is no ambiguity. With this in mind, the fluid four-velocity is given by

$$
u_{\mu}=a\left[-1-\Phi+\frac{1}{2} \Phi^{2}-\frac{1}{2} v_{(1)}^{j} v_{j}^{(1)}, v_{i}^{(1)}+\frac{1}{2}\left\{v_{i}^{(2)}-S_{i}\right\}-2 \Phi v_{i}^{(1)}\right]
$$

where $v_{i}^{(1)}=\partial_{i} v_{(1)}$ and $\partial_{i} v_{(2)}^{i}=0$.

The background dynamics are given by

$$
\mathcal{H}^{2}=\frac{8}{3} \pi G a^{2} \rho+\frac{1}{3} a^{2} \Lambda, \quad \mathcal{H}^{\prime}=-4 \pi G(1+w) a^{2} \rho+\mathcal{H}^{2},
$$

*Electronic address: teresa.huichinglu@gmail.com 
where $w=p / \rho=$ const (later we specialize to $w=0$ ). The first-order perturbed field equations lead to 8

$$
\begin{aligned}
v_{(1)} & =-\frac{\Phi^{\prime}+\mathcal{H} \Phi}{4 \pi G \rho a^{2}(1+w)}, \\
\delta \rho & =\frac{\nabla^{2} \Phi-3 \mathcal{H}\left(\Phi^{\prime}+\mathcal{H} \Phi\right)}{4 \pi G a^{2}},
\end{aligned}
$$

where the Newtonian potential obeys the evolution equation

$$
\Phi^{\prime \prime}+3 \mathcal{H}(1+w) \Phi^{\prime}+\left[(1+w) \Lambda a^{2}-w \nabla^{2}\right] \Phi=0 .
$$

All linear scalar modes are determined by $\Phi$, and terms quadratic in $\Phi$ and its derivatives will source the secondorder vector modes. Therefore we will require the power spectrum $\mathcal{P}_{\Phi}$. In the matter era $(w=0)$, neglecting $\Lambda$, we have $a=a_{0}\left(\eta / \eta_{0}\right)^{2}$, and the solution in Fourier space is $\Phi_{m}(k, \eta)=A_{m}(k)$, where we remove the decaying mode. Then the power spectrum today is computed using the growth suppression and transfer functions. (See Appendix A for more details.)

Vector perturbations typically produce vorticity and a transverse shear in the fluid four-velocity, Eq. 2). In order to compute them, we need the covariant definitions (see 9] for a recent review):

$$
\begin{aligned}
& \omega_{\mu \nu}=\delta^{(1)} \omega_{\mu \nu}+\delta^{(2)} \omega_{\mu \nu}=h_{[\mu}^{\alpha} h_{\nu]}^{\beta} u_{\alpha ; \beta}, \\
& \sigma_{\mu \nu}=\delta^{(1)} \sigma_{\mu \nu}+\delta^{(2)} \sigma_{\mu \nu}=\left\{h_{(\mu}^{\alpha} h_{\nu)}^{\beta}-\frac{1}{3} h_{\mu \nu} h^{\alpha \beta}\right\} u_{\alpha ; \beta},
\end{aligned}
$$

where $h_{\mu \nu}=g_{\mu \nu}+u_{\mu} u_{\nu}$ is the projector into the instantaneous fluid rest space, and $\omega_{\mu \nu} u^{\nu}=0=\sigma_{\mu \nu} u^{\nu}$. The vorticity is a purely vector quantity. The vector part of the second order shear is defined via (see Appendix D for how to get $\sigma_{j}$ from $\delta^{(2)} \sigma_{i j}$ )

$$
\delta^{(2)} \sigma_{i j}=a \partial_{(i} \sigma_{j)}, \quad \partial_{i} \sigma^{i}=0
$$

At first order, there is only scalar shear:

$$
\begin{aligned}
\delta^{(1)} \omega_{i j} & =0 \\
\delta^{(1)} \sigma_{i j} & =a\left(\partial_{i} \partial_{j}-\frac{1}{3} \delta_{i j} \nabla^{2}\right) v_{(1)} .
\end{aligned}
$$

\section{SECOND ORDER VECTOR MODES}

\section{A. Vorticity}

We use a covariant and fully nonlinear approach in this sub-section, which is more direct and transparent than a perturbative approach in the case of vorticity, and which also leads to a more general result.

The vorticity tensor of a fluid with four-velocity $u^{\mu}$ defines the vorticity vector $\omega_{\mu}=\varepsilon_{\mu \nu \gamma} \omega^{\nu \gamma} / 2$, where $\varepsilon_{\mu \nu \gamma}$ is the covariant permutation tensor in the fluid rest space. The vorticity vector obeys the following propagation equation, which is covariant and allows for full nonlinearity [9]:

$$
h_{\mu}{ }^{\nu} \dot{\omega}_{\nu}=-\frac{2}{3} \Theta \omega_{\mu}-\frac{1}{2} \operatorname{curl} \dot{u}_{\mu}+\sigma_{\mu \nu} \omega^{\nu} .
$$

Here $\Theta=\nabla_{\mu} u^{\mu}$ is the volume expansion rate, an overdot denotes covariant differentiation along the fluid flow $\left(u^{\mu} \nabla_{\mu}\right)$, so that $\dot{u}_{\mu}$ is the fluid four-acceleration, and the covariant spatial curl is defined by curl $n_{\mu}=\varepsilon_{\mu \nu \gamma} \mathrm{D}^{\nu} n^{\gamma}$, where $\mathrm{D}_{\mu}$ is the spatially projected covariant derivative $\left(\mathrm{D}_{\mu} n_{\nu}=h_{\mu}{ }^{\alpha} h_{\nu}{ }^{\beta} \nabla_{\alpha} n_{\beta}\right)$.

In order to evaluate the curl of the acceleration, we need the momentum conservation equation [9],

$$
h_{\mu}^{\nu} \dot{q}_{\nu}+\frac{4}{3} \Theta q_{\mu}+(\rho+p) \dot{u}_{\mu}+\mathrm{D}_{\mu} p+\mathrm{D}^{\nu} \pi_{\mu \nu}+\sigma_{\mu \nu} q^{\nu}-\varepsilon_{\mu \nu \gamma} \omega^{\nu} q^{\gamma}-\pi_{\mu \nu} \dot{u}^{\nu}=0
$$

where $q_{\mu}$ is the spatial momentum density flux relative to $u^{\mu}$ and $\pi_{\mu \nu}$ is the fluid anisotropic stress (spatial, tracefree and symmetric). In general, Eq. (13) shows that the curl of acceleration can introduce source terms for vorticity in 
Eq. (12). In the case of a perfect fluid however, we have $q_{\mu}=0=\pi_{\mu \nu}$ and $\mathrm{D}_{\mu} p=c_{s}^{2} \mathrm{D}_{\mu} \rho$, where $c_{s}$ is the adiabatic sound speed. Then using the exact identity 9 ]

$$
\operatorname{curl} \mathrm{D}_{\mu} f=-2 \dot{f} \omega_{\mu}
$$

the curl of Eq. (13) gives $(\rho+p) \operatorname{curl} \dot{u}_{\mu}=2 \dot{p} \omega_{\mu}+\varepsilon_{\mu \nu \gamma} \dot{u}^{\nu} \mathrm{D}^{\gamma}(\rho+p)$. Using Eq. 13) again, we find that the second term is proportional to $\varepsilon_{\mu \nu \gamma} \mathrm{D}^{\nu} p \mathrm{D}^{\gamma} \rho$, which vanishes, since $\mathrm{D}_{\mu} p$ is parallel to $\mathrm{D}_{\mu} \rho$ for a perfect fluid. Collecting results, we arrive at the fully nonlinear vorticity propagation equation for a perfect fluid:

$$
h_{\mu}{ }^{\nu} \dot{\omega}_{\nu}+\left(\frac{2}{3}-c_{s}^{2}\right) \Theta \omega_{\mu}-\sigma_{\mu \nu} \omega^{\nu}=0
$$

(Note that the last term on the left is at least third order.) This equation shows that there is no source for vorticity, so that vorticity cannot be generated in a perfect fluid, at any perturbative order.

In particular, there is no generation of vorticity at non-linear order by first-order scalar perturbations, in the case of a perfect fluid, and thus

$$
\delta^{(2)} \omega_{i j}=0
$$

If there is primordial vorticity, then it must be introduced as an initial condition. Any primordial vorticity will simply redshift away as the universe expands, according to Eq. 15), and will be entirely unaffected by the growth of density perturbations. Effectively, the density perturbations generate metric vector perturbations, and the fluid velocity adjusts so as to maintain zero vorticity. This is similar to what happens with the Harrison mechanism for magnetogenesis, where the vector modes generated at first order by defects cannot induce vorticity in the plasma [10]. For more than one perfect fluid, vorticity non-generation applies separately to each fluid, as long as there is no momentum exchange between the fluids. This is the case for example with cold dark matter and baryons, which interact only gravitationally.

Returning to the perturbative analysis, we can now use Eq. [16], together with the Einstein equations, to determine the vector metric perturbation $S^{i}$.

\section{B. Vector metric perturbations}

The second-order vorticity is given by Eqs. 22) and (7):

$$
\delta^{(2)} \omega_{i j}=\frac{a}{2}\left\{\partial_{[j} v_{i]}^{(2)}-\partial_{[j} S_{i]}+6 \partial_{[i} \Phi \partial_{j]} v^{(1)}+2 \partial_{[i} v^{(1) \prime} \partial_{j]} v^{(1)}\right\}
$$

which is in agreement with the expression in [11. Then Eq. (16) becomes a constraint on $v_{(2)}^{i}-S^{i}$. However, we have another constraint from the second order $0 i$ Einstein equation [7]

$$
6 \mathcal{H}^{2} \Omega_{m}(1+w)\left[v_{i}^{(2)}-S_{i}\right]=-\nabla^{2} S_{i}+8\left[2 \Phi^{\prime} \partial_{i} \Phi+\frac{2}{3 \mathcal{H}^{2} \Omega_{m}} \nabla^{2} \Phi \partial_{i}\left(\Phi^{\prime}+\mathcal{H} \Phi\right)\right]^{V},
$$

where $V$ denotes schematically the vector part of the quadratic source term which can be extracted in Fourier space as shown in Equation (C2). Substituting Eqs. (4) and (18) into Eq. (17), we obtain

$$
\delta^{(2)} \omega_{i j}=\frac{a}{12(1+w) \mathcal{H}^{2} \Omega_{m}}\left\{\nabla^{2} \partial_{[i} S_{j]}-\frac{16}{3(1+w) \mathcal{H}^{2} \Omega_{m}}\left(\nabla^{2} \partial_{[i} \Phi\right) \partial_{j]}\left(\Phi^{\prime}+\mathcal{H} \Phi\right)\right\} .
$$

Then Eqs. 16) and (19) imply that

$$
\nabla^{2} S_{i}=\frac{16}{3 \mathcal{H}^{2} \Omega_{m}(1+w)}\left\{\nabla^{2} \Phi \partial_{i}\left(\Phi^{\prime}+\mathcal{H} \Phi\right)\right\}^{V} .
$$

This directly recovers the solution that was obtained in Ref. [7] via a Fourier-space projection of the second-order $i j$ Einstein equation, i.e., the vector anisotropic stress constraint,

$$
\partial_{(i} S_{j)}^{\prime}+2 \mathcal{H} \partial_{(i} S_{j)}=a \partial_{(i} \pi_{j)} .
$$


Here $\pi_{j}$ (with $\partial_{j} \pi^{j}=0$ ) is the effective vector anisotropic stress from second-order density perturbations (see [7] and Appendix B).

To evaluate $S_{i}$ via Eq. 201, we work in Fourier space, and the details are given in Appendix C. Using the first-order power spectrum (Appendix A), the resulting second-order vector power spectrum $\mathcal{P}_{S}$ is shown in Fig. 1 This may be compared with the power spectrum in [7], which is computed from scalar modes in the radiation era $(w=1 / 3)$, assuming a power-law form for the scalar spectrum. Here we have found $\mathcal{P}_{S}$ today, and we have calculated $\mathcal{P}_{\Phi}$ directly from the first-order solutions, using the transfer function to relate back to the primordial perturbations and the growth suppression factor to take account of $\Lambda$ in the background.
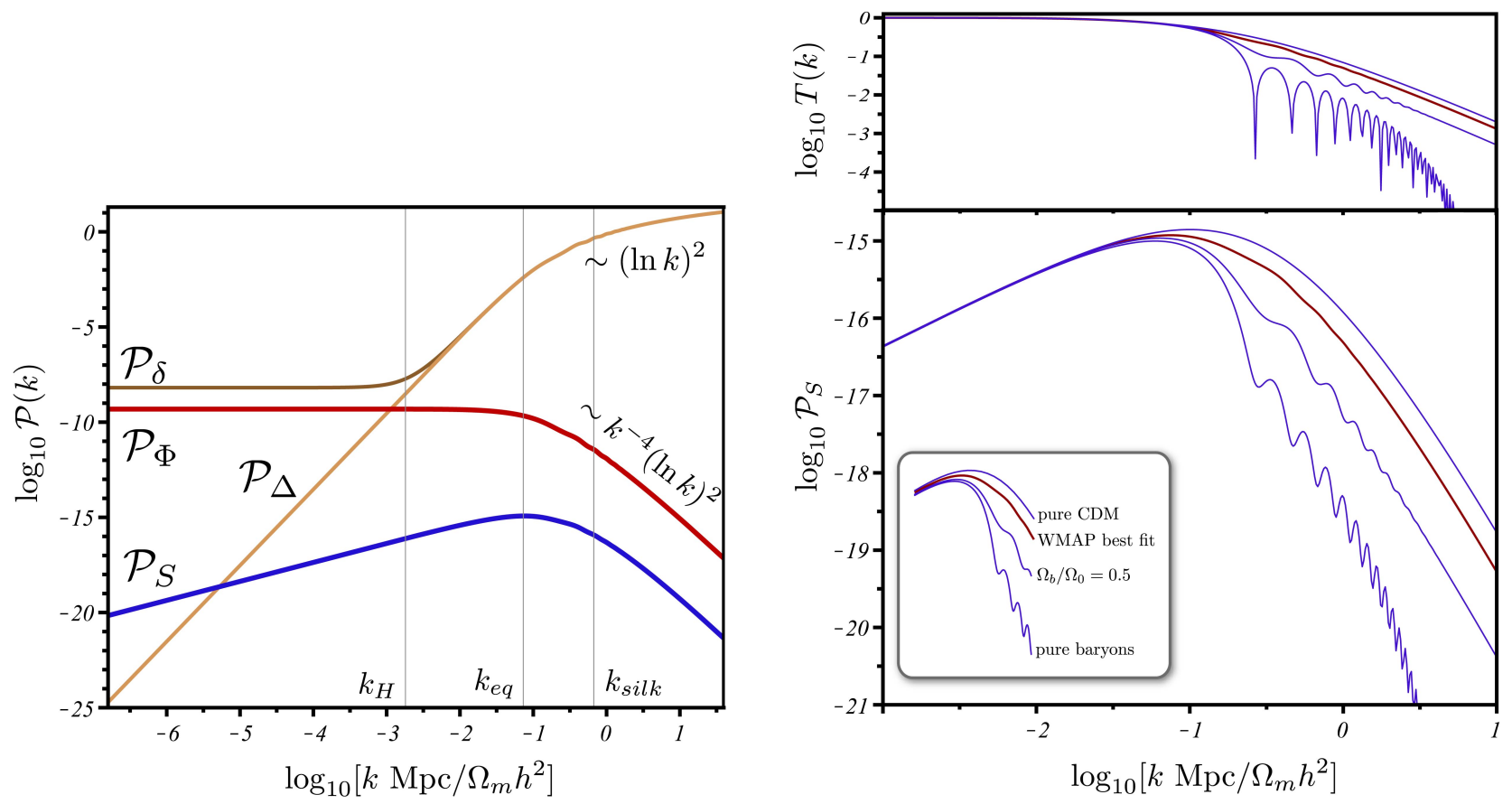

FIG. 1: The power spectrum today of metric vector modes generated at second order by density perturbations. Left: $\mathcal{P}_{S}$ together with the power spectra of first-order quantities: the density perturbation $\delta=\delta \rho / \rho$ and comoving density perturbation $\Delta=\delta-3 \mathcal{H} v^{(1)}$, and $\Phi$ (using best-fit WMAP5 parameters [12]: $\Omega_{m} h^{2}=0.1326,100 \Omega_{b} h^{2}=2.263, h=0.719$ ). Right: We show how increasing the baryon fraction decreases the power in the vectors above $k_{e q}$. The baryon oscillations are washed out to some extent in the vectors as can be seen by comparing $\mathcal{P}_{S}$ with the first-order transfer function in the top panel.

In Fig. 1 we compare the power spectrum of the vectors with that of the Newtonian potential. For comparison we also show the power spectrum of the two gauge-invariant density perturbations. The amplitude of the vectors decays on small scales, $k>k_{e q} \approx 0.009 \mathrm{Mpc}^{-1}$, in contrast to the density perturbation, which is growing, but in line with $\Phi$. For WMAP5 data 12 ]

$$
\mathcal{P}_{S} \approx 6.5 \times 10^{-5} \mathcal{P}_{\Phi} \text { for } k \gtrsim k_{\text {silk }} \approx 0.09 \mathrm{Mpc}^{-1}
$$

so that the amplitude of the metric vector modes is nearly $1 \%$ that of the metric scalar modes on small scales. For zero baryons we find that $\mathcal{P}_{S} \approx z_{\text {eq }}^{-1}\left(5.49 \Omega_{m} h^{2}-0.13\right)^{2.33} \mathcal{P}_{\Phi} \sim(\ln k)^{2} / k^{4}$ for $k \gtrsim k_{\text {silk }} \approx 0.09 \mathrm{Mpc}{ }^{-1}$. On large scales $\mathcal{P}_{S}$ scales like $k$, with a peak in the spectrum around the equality scale. This is analogous to the peak in the induced gravitational wave background on similar scales [13. In Fig. 11 we also show that including baryons induces oscillations in the vector power spectrum (bottom panel). They are washed out in comparison to the those present in the scalars (top panel), but are still very prominent.

The overall shape of the $S^{i}$ spectrum may be understood from the generation of vectors during the radiation era 7]. Vector modes grow outside the Hubble radius as $a^{1 / 2}$ only through the interaction of scalar modes which are larger than the Hubble radius. Inside the Hubble radius, vector modes decay, slightly less rapidly than $a^{-2}$, when fluctuations in the radiation fluid no longer support vectors. At the end of the radiation era, vector modes with $k<k_{e q}$ have acquired a tilt because modes are more aggressively produced by scalars which are close to the Hubble radius - very long wavelength modes interact only weakly. After equality, all vector modes grow at the same rate, so that those which entered the Hubble radius before equality are suppressed. 


\section{Vector Shear}

The second order vector shear follows from Eq. (8) as

$$
\delta^{(2)} \sigma_{i j}=\frac{a}{2} \partial_{(i} v_{j)}^{(2)}+a\left\{\partial_{(i} v^{(1)} \partial_{j)}\left[\Phi+v_{(1)}^{\prime}\right]\right\}^{V}=a \partial_{(i} \sigma_{j)} .
$$

Using Eqs. (3), (4) and (18), we have

$$
\begin{aligned}
a \partial_{(i} \sigma_{j)}= & \frac{a}{2} \partial_{(i}\left\{S_{j)}-\frac{1}{6 \mathcal{H}^{2} \Omega_{m}(1+w)} \nabla^{2} S_{j)}\right\} \\
& -\frac{2 a}{9 \mathcal{H}^{4} \Omega_{m}^{2}(1+w)^{2}}\left\{\mathcal{H}^{3}\left[2+3\left(2-\Omega_{m}\right)(1+w)\right] \partial_{i} \Phi \partial_{j} \Phi+2 \mathcal{H}(4+3 w) \partial_{i} \Phi^{\prime} \partial_{j} \Phi^{\prime}\right. \\
& \left.+2 \mathcal{H}^{2}\left[1+3\left(1+\Omega_{m}\right)(1+w)\right]\left(\partial_{i} \Phi \partial_{j} \Phi\right)^{\prime}-(1+2 w) \nabla^{2} \partial_{(i} \Phi \partial_{j)}\left(\Phi^{\prime}+\mathcal{H} \Phi\right)\right\}^{V} .
\end{aligned}
$$

In order to find the vector power spectrum $\mathcal{P}_{\sigma V}$ for $\sigma_{i}$, we substitute Eq. 20 into Eq. (24), and then apply the vector extraction operator in Fourier space. Further details are given in Appendix C. The resulting power spectrum has a similar shape to $\mathcal{P}_{S}$, since $\sigma_{i}=S_{i} / 2+$ small corrections by Eq. (24).

It is also useful to compare the first-order scalar and second-order vector contributions to the shear energy density. We define the dimensionless shear density

$$
\Omega_{\sigma}=\frac{a^{2}}{6 \mathcal{H}^{2}} \sigma_{\mu \nu} \sigma^{\mu \nu}
$$

The scalar and vector contributions to shear are given by $\sigma=v_{(1)}$ [Eq. (11)] and $\sigma_{i}$ [Eq. [24)] respectively. They define scalar power $\mathcal{P}_{\sigma S}$ and vector power $\mathcal{P}_{\sigma V}$, which then define the spatial averages of $\Omega_{\sigma S}, \Omega_{\sigma V}$ via

$$
\frac{d\left\langle\Omega_{\sigma S}\right\rangle}{d \ln k}=\frac{a^{2} k^{4}}{6 \mathcal{H}^{2}} \mathcal{P}_{\sigma S}, \quad \frac{d\left\langle\Omega_{\sigma V}\right\rangle}{d \ln k}=\frac{a^{2} k^{2}}{12 \mathcal{H}^{2}} \mathcal{P}_{\sigma V} .
$$

Note that there are 2 polarizations implicit in $\mathcal{P}_{\sigma V}$.

The quantities in Eq. (26) are shown in Fig. 2. As $k \rightarrow 0, d\left\langle\Omega_{\sigma V}\right\rangle / d \ln k \sim k^{3}$ and $d\left\langle\Omega_{\sigma S}\right\rangle / d \ln k \sim k^{4}$. This shows that the second-order vector shear is much smaller than the linear scalar shear - except on very large scales. However, this feature in the Poisson gauge will not lead to any growing physical effect, since both quantities are decaying on large scales.

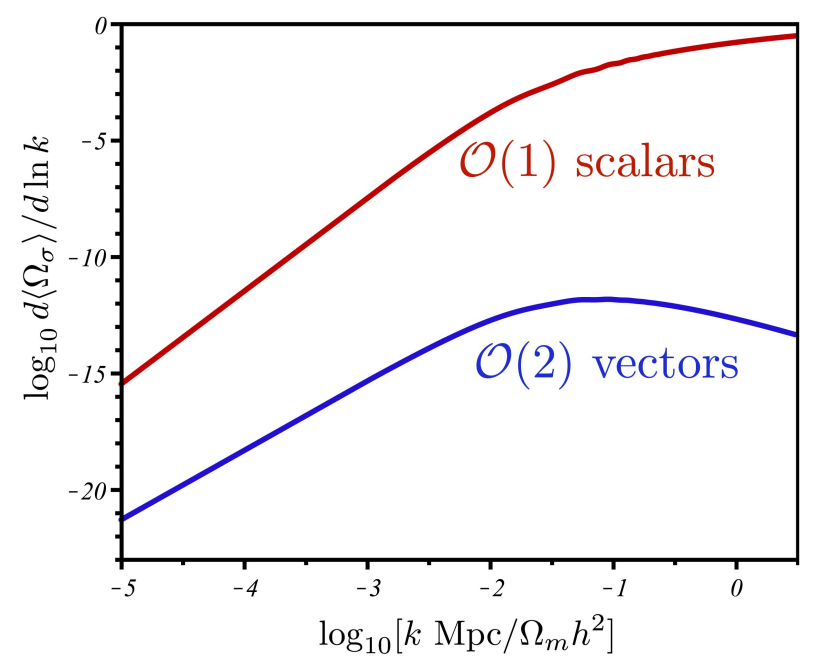

FIG. 2: The energy densities of the first-order scalar and second-order vector shear per logarithmic $k$-interval.

The vector part of the shear may be interpreted as a rotational quantity, even though the vorticity of the fluid is zero. We can see this readily via the covariant approach of section IIA. The vector shear is

$$
\sigma_{\mu \nu}=\mathrm{D}_{\langle\mu} \sigma_{\nu\rangle}:=\left\{h_{(\mu}^{\alpha} h_{\nu)}^{\beta}-\frac{1}{3} h_{\mu \nu} h^{\alpha \beta}\right\} \mathrm{D}_{\alpha} \sigma_{\beta}, \quad \mathrm{D}_{\mu} \sigma^{\mu}=0 .
$$


By boosting from the fluid rest frame to a different frame, $\tilde{u}^{\mu}=u^{\mu}+v^{\mu}$ (with $v_{\mu} u^{\mu}=0$ ), the vorticity and shear become 9 ]

$$
\tilde{\omega}_{\mu}=-\frac{1}{2} \operatorname{curl} v_{\mu}, \quad \tilde{\sigma}_{\mu}=\sigma_{\mu}+v_{\mu} .
$$

If we choose the frame by $v_{\mu}=-\sigma_{\mu}$, the vector part of the shear $\tilde{\sigma}_{\mu}$ is zero, but the vorticity $\tilde{\omega}_{\mu}$ no longer vanishes. This shows the essentially rotational nature of the fluid vector shear, even though the vorticity of the fluid is zero. Note that it is not possible to boost away the scalar or tensor part of the shear in this way.

\section{CONCLUSIONS}

We have computed the power spectra for the metric vector perturbations and vector shear at second order, generated by first-order scalar perturbations in a $\Lambda \mathrm{CDM}$ model. In addition, we used a covariant approach to show explicitly how vorticity is not generated in a perfect fluid at any perturbative order by first-order scalar perturbations, so that there is no vorticity in the matter. In order to generate vorticity, one requires either an imperfect fluid, or momentum exchange between the fluid and another fluid. Momentum exchange (via Compton and Coulomb interactions) between electrons, protons and photons in the radiation and recombination eras can generate vorticity and magnetic fields at second order [11, 14, 15, 16, 17, 18, 19].

In order to obtain the vector quantities $S^{i}$ and $v_{(2)}^{i}$, we used the vanishing of vorticity [Eq. [16]] and the $0 i$ Einstein constraint [Eq. [18]]. Alternatively, one could also use the $i j$ Einstein equation [Eq. [21]] and the momentum conservation equation, which has the form

$$
\left\{v_{(2) i}-S_{i}\right\}^{\prime}+\mathcal{H}\left(1-3 c_{s}^{2}\right)\left\{v_{(2) i}-S_{i}\right\}=M_{i},
$$

where the source term $M^{i}$ is given in Appendix B.

The cosmological background of vector modes is small, especially if measured in terms of the dimensionless shear density, as shown in Figs. 1 and 2. However, given that the amplitude of the vector modes in the metric is as large as $\sim 1 \%$ of the metric first-order scalar modes, in principle these vector modes will have an effect on various cosmological observations. In particular:

- Redshift-space distortions [20: the divergenceless velocity $v_{(2)}^{i}$ will make a contribution to radial peculiar velocities and thus to redshift-space distortions.

- Large-angle CMB temperature anisotropies [2, 5]: the vector modes will contribute to the Doppler and integrated Sachs-Wolfe effects:

$$
\delta^{(2)} T=\left.\frac{1}{2}\left\{v_{i}^{(2)}-S_{i}\right\} e^{i}\right|_{E} ^{O}+\frac{1}{2} \int_{E}^{O} d \lambda \partial_{i} S_{j} e^{i} e^{j}
$$

- Weak lensing [21, 22]: vector modes produce a deflection angle

$$
\vec{\alpha}=\int_{E}^{O} d \lambda(\vec{\nabla} \times \vec{S}) \times \vec{e} .
$$

- CMB polarization [4, 23]: the vector modes will leave a characteristic imprint on CMB polarization.

Further work is needed to compute the size of these vector corrections. They are likely to be significant mainly below $\sim 10 \mathrm{Mpc}$, but in this regime the scalar non-linear effects are important and likely to dominate.

The vector degree of freedom forms an integral part of the perturbative expansion when one goes beyond linear order. At the order we have considered, vectors must be present essentially through a constraint in the field equations arising at order $\Phi^{2}$, even though vectors have no independent propagating degrees of freedom. This is distinct from the intrinsically propagating degree of freedom in the scalar-induced gravitational wave background [13, 24, 25. We have shown that the vector mode background has maximum power around the equality scale, similar to the induced gravitational wave background, and the metric vector modes achieve their maximal fraction of the linear metric scalar modes on scales below the Silk scale. The size of the vector contribution to the full non-linear power spectrum relevant for structure formation remains to be calculated. 


\section{Acknowledgements:}

We thank Bruce Bassett, Marco Bruni, Ruth Durrer, Cyril Pitrou, Jean-Phillipe Uzan and David Wands for useful discussions and comments. THCL, KA and CC are supported by the National Research Foundation (South Africa), and RM is supported by STFC (UK). THCL and CC acknowledge financial support from the University of Cape Town. KA is additionally supported by the Italian Ministero Degli Affari Esteri-DG per la Promozione e Cooperazione Culturale under the joint Italy/ South Africa Science and Technology agreement. THCL, KA and CC thank the ICG Portsmouth for hospitality during various stages of the work; their visits were partly supported by a joint Royal Society (UK) - National Research Foundation (South Africa) grant UID 65329.

\section{APPENDIX A: FIRST-ORDER SCALAR POWER SPECTRUM}

The power spectrum for the first-order scalar perturbations is defined by

$$
\left\langle\Phi^{*}(\boldsymbol{k}, \eta) \Phi\left(\boldsymbol{k}^{\prime}, \eta\right)\right\rangle=\frac{2 \pi^{2}}{k^{3}} \delta^{3}\left(\boldsymbol{k}-\boldsymbol{k}^{\prime}\right) \mathcal{P}_{\Phi}(k, \eta) .
$$

In the early radiation era,

$$
\mathcal{P}_{\Phi_{r}}(k) \approx A_{r}(k)^{2} \frac{k^{3}}{486 \pi^{2}}, \quad A_{r}(k)^{2} \approx \frac{216 \pi^{2}}{k^{3}} \Delta_{\mathcal{R}}^{2}(k),
$$

where $\Delta_{\mathcal{R}}^{2}$ is the primordial power of the curvature perturbation, with 12 . $\Delta_{\mathcal{R}}^{2} \approx 2.41 \times 10^{-9}$ at a scale $k_{C M B}=$ $0.002 \mathrm{Mpc}^{-1}$. By conservation of the curvature perturbation, $\Phi_{m}=9 \Phi_{r} / 10$ at equality, so the early matter power is given by

$$
\tilde{A}_{m}(k)=\frac{\sqrt{3}}{30 g_{\infty}} A_{r}(k) \approx \frac{3 \sqrt{2} \pi \Delta_{\mathcal{R}}}{5 g_{\infty} k^{3 / 2}}
$$

assuming a scale-invariant initial spectrum. Here $g_{\infty}$ is a normalization parameter in the $\Lambda$ growth suppression function

$$
g(z)=\frac{5}{2} g_{\infty} \Omega_{m}(z)\left\{\Omega_{m}(z)^{4 / 7}-\Omega_{\Lambda}(z)+\left[1+\frac{1}{2} \Omega_{m}(z)\right]\left[1+\frac{1}{70} \Omega_{\Lambda}(z)\right]\right\}^{-1}
$$

and $g_{\infty}$ is chosen so that $g(0)=1$. The power today is given by $A_{m}(k)=\tilde{A}_{m}(k) T(k)$, where $T(k)$ is the normalized transfer function [26] (see Fig. 11):

$$
\mathcal{P}_{\Phi}=\left(\frac{3 \Delta_{\mathcal{R}}}{5 g_{\infty}}\right)^{2} g^{2} T(k)^{2}
$$

\section{APPENDIX B: VECTOR EVOLUTION AND MOMENTUM CONSERVATION}

The source term in the vector evolution equation Eq. 210 is as follows (where we assume $w=$ const):

$$
a \partial_{(i} \pi_{j)}=4\left[1+\frac{2}{3 \Omega_{m}(1+w)}\right]\left\{\partial_{i} \Phi \partial_{j} \Phi\right\}^{V}+\frac{8}{3 \mathcal{H}^{2} \Omega_{m}(1+w)}\left\{\partial_{i} \Phi^{\prime} \partial_{j} \Phi^{\prime}+2 \mathcal{H} \partial_{(i} \Phi \partial_{j)} \Phi^{\prime}\right\}^{V}
$$

where $V$ denotes the vector part, and we have used the fact that $\left\{\Phi \partial_{i} \partial_{j} \Phi\right\}^{V}=-\left\{\partial_{i} \Phi \partial_{j} \Phi\right\}^{V}$.

In Eq. 29), the source term in the vector momentum conservation equation is as follows (where we also assume $w=$ const): 


$$
\begin{aligned}
9 \mathcal{H}^{4} \Omega_{m}^{2}(1+w)^{2} M_{i}= & 6 \mathcal{H}^{3}\left[24\left(1+c_{s}^{2}\right)^{2}-12\left(1+c_{s}^{2}\right)(2+w)+\Omega_{m}(1+w)\left(5+18 c_{s}^{2}-15 w\right)\right]\left\{\Phi \partial_{i} \Phi^{\prime}\right\}^{V} \\
& +6 \mathcal{H}^{3}\left[24\left(1+c_{s}^{2}\right)^{2}-12\left(1+c_{s}^{2}\right)(2+w)+2 \Omega_{m}(1+w)\left(3 c_{s}^{2}-2\right)\right]\left\{\Phi^{\prime} \partial_{i} \Phi\right\}^{V} \\
& +2 \mathcal{H}\left[10\left(1+c_{s}^{2}\right)-18\left(1+c_{s}^{2}\right)^{2}-6\left(1+c_{s}^{2}\right)(1+w)-4\right]\left\{\mathcal{H} \partial_{i} \Phi \nabla^{2} \Phi+\partial_{i} \Phi^{\prime} \nabla^{2} \Phi\right\}^{V} \\
& -36 \mathcal{H}^{2} c_{s}^{2}\left[2\left(1+c_{s}^{2}\right)+\Omega_{m}(1+w)\right]\left\{\Phi \nabla^{2} \partial_{i} \Phi\right\}^{V}+8 c_{s}^{2}\left\{\mathcal{H} \nabla^{2} \Phi^{\prime} \partial_{i} \Phi+\nabla^{2} \Phi^{\prime} \partial_{i} \Phi^{\prime}\right\}^{V} \\
& +4\left(1+c_{s}^{2}\right) c_{s}^{2}\left\{\nabla^{2} \Phi \nabla^{2} \partial_{i} \Phi\right\}^{V}-24 \mathcal{H}\left(1+c_{s}^{2}\right) c_{s}^{2}\left\{\Phi^{\prime} \nabla^{2} \partial_{i} \Phi\right\}^{V} \\
& +8 \mathcal{H}\left\{\partial^{k} \Phi^{\prime} \partial_{k} \partial_{i} \Phi+\partial^{k} \Phi \partial_{k} \partial_{i} \Phi^{\prime}\right\}^{V} .
\end{aligned}
$$

\section{APPENDIX C: VECTOR MODE POWER SPECTRUM}

We define the Fourier transform of the vector perturbation as

$$
S_{i}(\boldsymbol{x}, \eta)=\frac{1}{(2 \pi)^{3 / 2}} \int d^{3} k\left[S(\boldsymbol{k}, \eta) e_{i}(\boldsymbol{k})+\bar{S}(\boldsymbol{k}, \eta) \bar{e}_{i}(\boldsymbol{k})\right] e^{i \boldsymbol{k} \cdot \boldsymbol{x}},
$$

where the two orthonormal basis vectors $\boldsymbol{e}$ and $\overline{\boldsymbol{e}}$ are orthogonal to $\boldsymbol{k}$ with

$$
S(\boldsymbol{k}, \eta)=\frac{1}{(2 \pi)^{3 / 2}} \int d^{3} x S_{i}(\boldsymbol{x}, \eta) e^{i}(\boldsymbol{k}) e^{-i \boldsymbol{k} \cdot \boldsymbol{x}} .
$$

The inverse integral is similarly defined for its second parity $\bar{S}(\boldsymbol{k}, \eta)$. Equation 20 gives a solution for $S(\boldsymbol{k}, \eta)$ in Fourier space

$$
S(\boldsymbol{k}, \eta)=\frac{16 i}{3 \Omega_{m}(2 \pi)^{3 / 2}} \frac{e^{j}(\boldsymbol{k})}{k^{2}} \int d^{3} k^{\prime}\left|\boldsymbol{k}-\boldsymbol{k}^{\prime}\right|^{2} k_{j}^{\prime} \mathcal{B}\left(\boldsymbol{k}-\boldsymbol{k}^{\prime}, \boldsymbol{k}^{\prime}, \eta\right)
$$

where

$$
\mathcal{B}\left(\boldsymbol{k}_{1}, \boldsymbol{k}_{2}, \eta\right)=\mathcal{H}^{-2} \Phi\left(\boldsymbol{k}_{1}, \eta\right)\left[\Phi^{\prime}\left(\boldsymbol{k}_{2}, \eta\right)+\mathcal{H} \Phi\left(\boldsymbol{k}_{2}, \eta\right)\right] .
$$

Defining the power spectrum as

$$
\left\langle S^{*}(\boldsymbol{k}, \eta) S\left(\boldsymbol{k}^{\prime}, \eta\right)\right\rangle=\frac{2 \pi^{2}}{k^{3}} \delta^{3}\left(\boldsymbol{k}-\boldsymbol{k}^{\prime}\right) \mathcal{P}_{S}(k, \eta) .
$$

we find, using Wick's theorem,

$$
\begin{aligned}
\mathcal{P}_{S}(k, \eta) & =\frac{16}{9 k \Omega_{m}^{2} \pi^{5}} \int d^{3} k^{\prime}\left|\boldsymbol{k}-\boldsymbol{k}^{\prime}\right|^{2}\left[k_{j}^{\prime} e^{j}(\boldsymbol{k})\right]\left[k_{m}^{\prime} e^{m}(\boldsymbol{k})\right] \mathcal{B}\left(\left|\boldsymbol{k}-\boldsymbol{k}^{\prime}\right|, k^{\prime}, \eta\right) \\
& \times\left\{\left|\boldsymbol{k}-\boldsymbol{k}^{\prime}\right|^{2} \mathcal{B}\left(\left|\boldsymbol{k}-\boldsymbol{k}^{\prime}\right|, k^{\prime}, \eta\right)-\left(k^{\prime}\right)^{2} \mathcal{B}\left(k^{\prime},\left|\boldsymbol{k}-\boldsymbol{k}^{\prime}\right|, \eta\right)\right\}
\end{aligned}
$$

This may be simplified to give

$$
\mathcal{P}_{S}(k)=\left(\frac{2 \Delta_{\mathcal{R}}}{5 g_{\infty}}\right)^{4}\left(\frac{3 g\left[g^{\prime}+\mathcal{H} g\right]}{\Omega_{m} \mathcal{H}^{2}}\right)^{2} k^{2} \Pi\left(u^{2}\right)
$$

where

$$
\Pi(\xi)=\int_{0}^{\infty} d v \int_{|v-1|}^{v+1} d u \xi(u v)^{-2}\left(u^{2}-v^{2}\right)\left[4 v^{2}-\left(1+v^{2}-u^{2}\right)^{2}\right][T(k v) T(k u)]^{2},
$$

and $v=k^{\prime} / k, u=\sqrt{1+v^{2}-2 v \cos \theta}$ and $\cos \theta=\boldsymbol{k}^{\prime} \cdot \boldsymbol{k} /\left(k^{\prime} k\right)$. 


\section{APPENDIX D: VECTOR SHEAR POWER SPECTRUM}

To extract the divergenceless vector $\sigma_{i}$ from $\partial_{(i} \sigma_{j)}$, we use the operator $\mathcal{V}_{m}^{i j}[7$

$$
\mathcal{V}_{m}^{i j}\left(\boldsymbol{x}, \boldsymbol{x}^{\prime}\right)=-\frac{2 i}{(2 \pi)^{3}} \int d^{3} k^{\prime} k^{\prime-2} \int d^{3} x^{\prime} k^{i}\left[e_{m}\left(\boldsymbol{k}^{\prime}\right) e^{j}\left(\boldsymbol{k}^{\prime}\right)+\bar{e}_{m}\left(\boldsymbol{k}^{\prime}\right) \bar{e}^{j}\left(\boldsymbol{k}^{\prime}\right)\right] e^{i \boldsymbol{k}^{\prime} \cdot\left(\boldsymbol{x}-\boldsymbol{x}^{\prime}\right)}
$$

Then $\sigma_{m}(\boldsymbol{x})=\mathcal{V}_{m}^{i j}\left(\boldsymbol{x}, \boldsymbol{x}^{\prime}\right) \partial_{(i} \sigma_{j)}\left(\boldsymbol{x}^{\prime}\right)$, and Eqs. 200 and (24) lead to the second-order vector shear in Fourier space

$$
\begin{aligned}
\sigma(\boldsymbol{k}, \eta)= & \frac{-2 a i}{3 \Omega_{m}(2 \pi)^{3 / 2}} \frac{e^{j}(\boldsymbol{k})}{k^{2}} \int d^{3} k^{\prime} k_{j}^{\prime}\left\{\left(k^{2}-6\left(k_{i}^{\prime} k^{i}\right)-4\left|\boldsymbol{k}-\boldsymbol{k}^{\prime}\right|^{2}\right) \mathcal{B}\left(\boldsymbol{k}-\boldsymbol{k}^{\prime}, \boldsymbol{k}^{\prime}, \eta\right)\right. \\
& \left.+\frac{2}{3 \Omega_{m}}\left(k^{2}-2 k_{i}^{\prime} k^{i}\right)\left[\mathcal{C}\left(\boldsymbol{k}^{\prime}, \boldsymbol{k}-\boldsymbol{k}^{\prime}, \eta\right)+\left(1-\frac{3 \Omega_{m}}{2}\right) \mathcal{B}\left(\boldsymbol{k}^{\prime}, \boldsymbol{k}-\boldsymbol{k}^{\prime}, \eta\right)\right]\right\},
\end{aligned}
$$

where

$$
\mathcal{C}\left(\boldsymbol{k}_{1}, \boldsymbol{k}_{2}, \eta\right)=\frac{1}{\mathcal{H}^{3}} \Phi^{\prime}\left(\boldsymbol{k}_{1}, \eta\right)\left[\Phi^{\prime}\left(\boldsymbol{k}_{2}, \eta\right)+\mathcal{H} \Phi\left(\boldsymbol{k}_{2}, \eta\right)\right] .
$$

The power spectrum is defined as in Eq. (C4). By Eq. (D2) and Wick's theorem, we obtain

$$
\begin{aligned}
\mathcal{P}_{\sigma V}(k, \eta)= & \left(\frac{\Delta_{\mathcal{R}}}{g_{\infty}}\right)^{4}\left[\frac{3 k a\left(g^{\prime}+\mathcal{H} g\right)}{50 \Omega_{m} \mathcal{H}^{2}}\right]^{2}\left\{-2 g^{2} \Pi\left(2+u^{2}+3 v^{2}\right)-\frac{8}{3 \mathcal{H} \Omega_{m}} g\right. \\
& \left.\times\left[g^{\prime}+\left(1-\frac{3 \Omega_{m}}{2}\right) \mathcal{H} g\right] \Pi\left(1+2 v^{2}\right)+\frac{8}{9 \mathcal{H}^{2} \Omega_{m}^{2}}\left[g^{\prime}+\left(1-\frac{3 \Omega_{m}}{2}\right) \mathcal{H} g\right]^{2} \Pi\left(u^{2}-v^{2}\right)\right\},
\end{aligned}
$$

where $g$ is given by Eq. (A4) and $\Pi$ by Eq. (C7).

[1] S. Matarrese, S. Mollerach and M. Bruni, Phys. Rev. D 58, 043504 (1998) arXiv:astro-ph/9707278.

[2] S. Mollerach and S. Matarrese, Phys. Rev. D 56, 4494 (1997) arXiv:astro-ph/9702234.

[3] H. Noh and J. c. Hwang, Phys. Rev. D 69, 104011 (2004), arXiv:astro-ph/0305123.

[4] S. Mollerach, D. Harari and S. Matarrese, Phys. Rev. D 69, 063002 (2004) arXiv:astro-ph/0310711.

[5] K. Tomita, Phys. Rev. D 71, 083504 (2005) arXiv:astro-ph/0501663.

[6] F. C. Mena, D. J. Mulryne and R. Tavakol, Class. Quant. Grav. 24, 2721 (2007) arXiv:gr-qc/0702064.

[7] T. C. Lu, K. Ananda and C. Clarkson, Phys. Rev. D 77, 043523 (2008) arXiv:0709.1619 [astro-ph]].

[8] H. Kodama, and M. Sasaki, Prog. Theor. Phys. Suppl. 78, 1 (1984).

[9] C. G. Tsagas, A. Challinor and R. Maartens, Phys. Rept. 465, 61 (2008) arXiv:0705.4397 [astro-ph]].

[10] L. Hollenstein, C. Caprini, R. Crittenden and R. Maartens, Phys. Rev. D 77, 063517 (2008) arXiv:0712.1667 [astro-ph]].

[11] S. Matarrese, S. Mollerach, A. Notari and A. Riotto, Phys. Rev. D 71, 043502 (2005) arXiv:astro-ph/0410687.

[12] E. Komatsu et al. [WMAP5 Collaboration], arXiv:0803.0547 [astro-ph].

[13] D. Baumann, P. J. Steinhardt, K. Takahashi and K. Ichiki, Phys. Rev. D 76, 084019 (2007) arXiv:hep-th/0703290.

[14] R. Gopal and S. Sethi, Mon. Not. Roy. Astron. Soc. 363, 521 (2005) arXiv:astro-ph/0411170.

[15] K. Takahashi, K. Ichiki, H. Ohno and H. Hanayama, Phys. Rev. Lett. 95, 121301 (2005) arXiv:astro-ph/0502283.

[16] K. Ichiki, K. Takahashi, H. Ohno, H. Hanayama and N. Sugiyama, arXiv:astro-ph/0603631.

[17] E. R. Siegel and J. N. Fry, Astrophys. J. 651, 627 (2006) arXiv:astro-ph/0604526.

[18] T. Kobayashi, R. Maartens, T. Shiromizu and K. Takahashi, Phys. Rev. D 75, 103501 (2007) arXiv:astro-ph/0701596.

[19] S. Maeda, S. Kitagawa, T. Kobayashi and T. Shiromizu, arXiv:0805.0169 [astro-ph].

[20] R. E. Smith, R. K. Sheth and R. Scoccimarro, Phys. Rev. D 78, 023523 (2008) arXiv:0712.0017] [astro-ph]].

[21] P. Schneider, J. Ehlers and E. E. Falco, Gravitational Lenses (Springer-Verlag Berlin, 1992).

[22] R. Durrer, Phys. Rev. Lett. 72, 3301 (1994) arXiv:astro-ph/9401033.

[23] M. Kamionkowski, A. Kosowsky and A. Stebbins, Phys. Rev. Lett. 78, 2058 (1997) arXiv:astro-ph/9609132.

[24] K. N. Ananda, C. Clarkson, and D. Wands Phys. Rev. D 75, 123518 (2007) arXiv:gr-qc/0612013.

[25] A. Mangilli, N. Bartolo, S. Matarrese and A. Riotto, Phys. Rev. D 78, 083517 (2008) arXiv:0805.3234.

[26] D. J. Eisenstein, and W. Hu, Astrophys. J. 496, 605 (1998). 